\title{
Consistency and Robustness Properties of Support Vector Machines for Heavy-Tailed Distributions
}

\section{Arnout Van Messem Andreas Christmann}
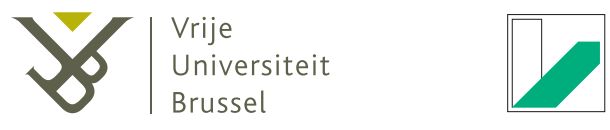

\section{UNIVERSITÄT}

BAYREUTH

\section{PhD Research Day VUB, May 28, 2010}




\section{Notation}

\section{Assumptions:}

- $\mathcal{X} \subseteq \mathbb{R}^{d}$ closed, $\mathcal{Y} \subseteq \mathbb{R}$ closed, $\mathcal{X} \neq \varnothing, \mathcal{Y} \neq \varnothing$

- $D=\left(\left(x_{1}, y_{1}\right), \ldots,\left(x_{n}, y_{n}\right)\right) \in(\mathcal{X} \times \mathcal{Y})^{n}, 1 \leq i \leq n$

- $\left(X_{i}, Y_{i}\right)$ i.i.d. $\sim \mathrm{P} \in \mathcal{M}_{1}(\mathcal{X} \times \mathcal{Y})$, $\mathrm{P}$ (totally) unknown $\hookrightarrow \mathrm{P}_{X}$ on $\mathcal{X}, \mathrm{P}(y \mid x)$ on $\mathcal{Y}$

Aim:

- $f(x)=$ quantity of interest

e.g., conditional median for robust regression

\section{Assumption:}

- Loss function $L: \mathcal{X} \times \mathcal{Y} \times \mathbb{R} \rightarrow[0, \infty), L(x, y, f(x))$ 


\section{Loss functions for regression}

eps-insensitive, eps $=0.5$

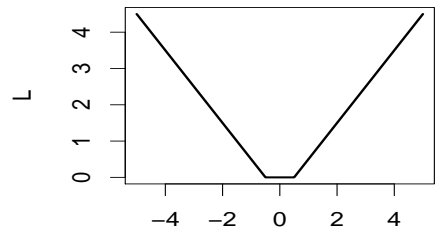

\section{$\stackrel{r}{r}$ Logistic}

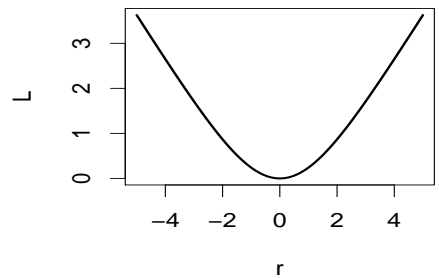

Huber, $c=1$

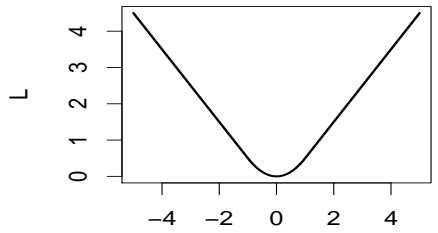

Pinball, tau $=0.10$

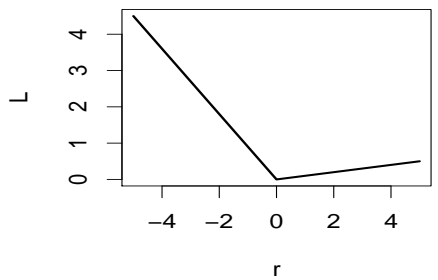




\section{Support Vector Machines (SVMs)}

Definition

$$
f_{L, \mathrm{P}, \lambda}:=\arg \inf _{f \in \mathcal{H}} \mathbb{E}_{\mathrm{P}} L(X, Y, f(X))+\lambda\|f\|_{\mathcal{H}}^{2}
$$

- $Y_{i} \mid x_{i}$ depends on an unknown function $f: \mathcal{X} \rightarrow \mathbb{R}$

- RKHS $\mathcal{H} \rightleftarrows$ kernel $k: \mathcal{X} \times \mathcal{X} \rightarrow \mathbb{R}, k$ measurable

- $\lambda>0$ regularization parameter

- $f_{L, \mathrm{D}, \lambda}:=\arg \min _{f \in \mathcal{H}} \frac{1}{n} \sum_{i=1}^{n} L\left(Y_{i}, f\left(X_{i}\right)\right)+\lambda\|f\|_{\mathcal{H}}^{2}$,

where $\mathrm{D}$ is empirical distribution for data set $D$ 


\section{Support Vector Machines}

\section{Notions}

- $L$ is called convex, continuous, Lipschitz continuous, differentiable, if $L$ has this property w.r.t. $3^{\text {rd }}$ argument

- $k$ is called bounded, if $\|k\|_{\infty}:=\sqrt{\sup _{x \in \mathcal{X}} k(x, x)}<\infty$

$$
\text { e.g. Gaussian RBF: } k\left(x, x^{\prime}\right)=e^{-\gamma\left\|x-x^{\prime}\right\|_{2}^{2}}, \gamma>0
$$

- $\Phi: \mathcal{X} \rightarrow \mathcal{H}, \Phi(x):=k(\cdot, x)$, is called canonical feature map

- Reproducing property:

$$
f(x)=\langle f, k(x, \cdot)\rangle_{\mathcal{H}} \quad \forall f \in \mathcal{H}, \forall x \in \mathcal{X} .
$$




\section{Example for feature map $\Phi(\mathrm{x})=\mathrm{k}(\mathrm{x}, \cdot)$}
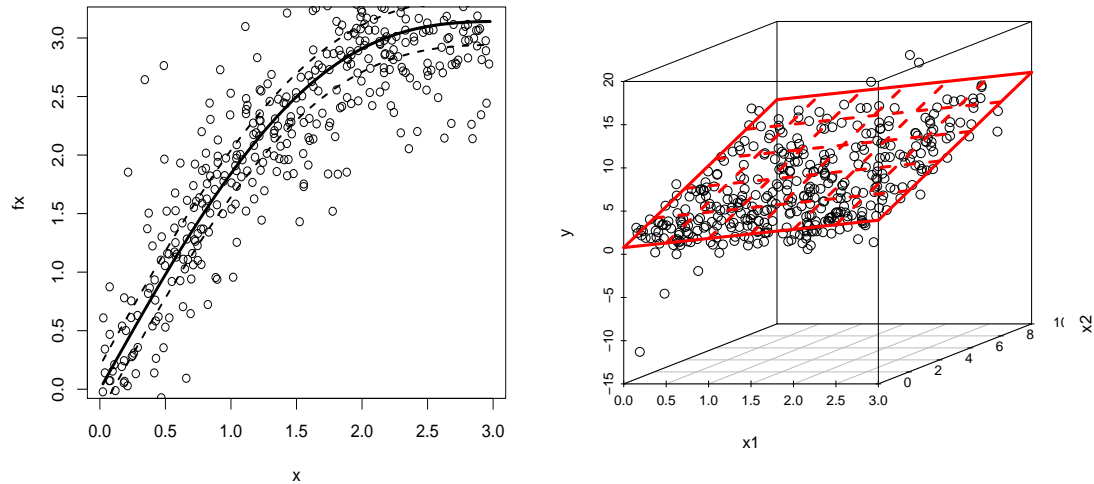


\section{Risk}

\section{Definitions}

Risk

Bayes risk

Bayes function $f_{L, \mathrm{P}}^{*}$
$\mathcal{R}_{L, \mathrm{P}}(f) \quad \mathbb{E}_{\mathrm{P}} L(X, Y, f(X))$

$\mathcal{R}_{L, \mathrm{P}}^{*}$ $\inf _{f: \mathcal{X} \rightarrow \mathbb{R} \text { measurable }} \mathcal{R}_{L, \mathrm{P}}(f)$

$\arg \inf _{f: \mathcal{X} \rightarrow \mathbb{R} \text { measurable }} \mathcal{R}_{L, \mathrm{P}}(f)$

\section{Questions}

Under which conditions on $\mathcal{X}, \mathcal{Y}, L, \mathcal{H}$, and $k$ do we have:

(1) $f_{L, P, \lambda}$ : existence, uniqueness

(2) Universal consistency to Bayes risk/function, i.e., $\forall \mathrm{P}$

$$
\begin{array}{r}
\mathcal{R}_{L, \mathrm{P}}\left(f_{L, \mathrm{D}, \lambda}\right) \stackrel{\mathrm{P}}{\longrightarrow} \mathcal{R}_{L, \mathrm{P}}^{*} \text { for }|D|=n \rightarrow \infty \\
f_{L, \mathrm{D}, \lambda} \stackrel{\mathrm{P}}{\longrightarrow} f_{L, \mathrm{P}}^{*} \text { for }|D|=n \rightarrow \infty
\end{array}
$$

(3) Robustness of $f_{L, \mathrm{P}, \lambda}$ ? 


\section{Known}

Support Vector Machines are consistent and robust, if based on Lipschitz continuous loss and bounded kernel.

Christmann \& Van Messem '08 Steinwart \& Christmann '08

Christmann \& Steinwart '07

\section{Question}

Can the assumptions $f \in L_{1}\left(\mathrm{P}_{X}\right)$ and $\int|Y| d \mathrm{P}<\infty$ be weakened?

(both for regression and classification problems)

$f \in L_{1}\left(\mathrm{P}_{X}\right) \quad$ if $\quad \int_{\mathcal{X}}|f(x)| d \mathrm{P}_{X}(x)<\infty$ 


\section{Shifted loss function}

Loss function $L: \mathcal{X} \times \mathcal{Y} \times \mathbb{R} \rightarrow[0, \infty)$ measurable

\section{Definition}

$$
\begin{aligned}
& L^{\star}: \mathcal{X} \times \mathcal{Y} \times \mathbb{R} \rightarrow \mathbb{R} \text { with } \\
& \qquad L^{\star}(x, y, t):=L(x, y, t)-L(x, y, 0) .
\end{aligned}
$$

Huber, 1967

$L^{\star}$ can be negative!

\section{Properties}

- $L$ (strictly) convex, then $L^{\star}$ (strictly) convex.

- $L$ Lipschitz continuous, then $L^{\star}$ Lipschitz continuous. 


\section{Shifted loss function}

\section{Conditions for finite risk}

For $L$ Lipschitz continuous

- $\mathcal{R}_{L, \mathrm{P}}(f)<\infty$ if $f \in L_{1}\left(\mathrm{P}_{X}\right)$ and $\mathbb{E}_{\mathrm{P}}|Y|<\infty$.

- $\mathcal{R}_{L^{\star}, \mathrm{P}}(f)<\infty$ if $f \in L_{1}\left(\mathrm{P}_{X}\right)$.

\section{Equality of SVMs}

If $f_{L, \mathrm{P}, \lambda}$ exists, then $f_{L^{\star}, \mathrm{P}, \lambda}=f_{L, \mathrm{P}, \lambda}$. 


\section{Existence and Uniqueness of SVM solution}

\section{Uniqueness}

- $L$ convex and $\mathcal{R}_{L^{\star}, \mathrm{P}}(f)<\infty$ for some $f \in \mathcal{H}$ and $\mathcal{R}_{L^{\star}, \mathrm{P}}(f)>-\infty$ for all $f \in \mathcal{H}$ OR

- $L$ is convex, Lipschitz continuous and $f \in L_{1}\left(\mathrm{P}_{X}\right)$.

Then, for all $\lambda>0$, there exists at most one SVM $f_{L^{\star}, \mathrm{P}, \lambda}$.

\section{Existence}

- $L$ convex, Lipschitz continuous,

- $\mathcal{H}$ RKHS of a bounded measurable kernel $k$.

Then, for all $\mathrm{P} \in \mathcal{M}_{1}(\mathcal{X} \times \mathcal{Y})$ and for all $\lambda>0$, there exists an SVM solution $f_{L^{\star}, \mathrm{P}, \lambda}$. 


\section{Consistency}

\section{Theorem}

- $L$ convex, Lipschitz continuous loss function,

- $\mathcal{H}$ RKHS of a bounded, measurable kernel $k$,

- $\left(\lambda_{n}\right)$ sequence of strictly positive numbers with $\lambda_{n} \rightarrow 0$.

Then, for all $\mathrm{P} \in \mathcal{M}_{1}(\mathcal{X} \times \mathcal{Y})$ and all $D$ with $|D|=n$,

(1) if $\lambda_{n}^{2} n \rightarrow \infty$, then $\mathcal{R}_{L^{\star}, \mathrm{P}}\left(f_{L^{\star}, \mathrm{D}, \lambda_{n}}\right) \stackrel{\mathrm{P}}{\longrightarrow} \mathcal{R}_{L^{\star}, \mathrm{P}}^{*}$.

(2) if $\lambda_{n}^{2+\delta} n \rightarrow \infty$ for some $\delta \in(0, \infty)$, then

$$
\mathcal{R}_{L^{\star}, \mathrm{P}}\left(f_{L^{\star}, \mathrm{D}, \lambda_{n}}\right) \stackrel{\text { a.s. }}{\longrightarrow} \mathcal{R}_{L^{\star}, \mathrm{P}}^{*} \text {. }
$$

(3) if $L=L_{\tau}$ pinball loss: $d\left(f_{L^{\star}, \mathrm{D}, \lambda_{n}}, f_{L_{\tau}, \mathrm{P}}^{*}\right) \rightarrow 0$. $d$ is a metric describing convergence in probability. 


\section{Robustness}

(1) What if $\left(X_{i}, Y_{i}\right)$ i.i.d. $\sim \mathrm{P}, \mathrm{P} \in \mathcal{M}_{1}$ unknown is invalid?

(2) What is the impact on $S: \mathrm{P} \mapsto f_{L^{\star}, \mathrm{P}, \lambda}$ ?

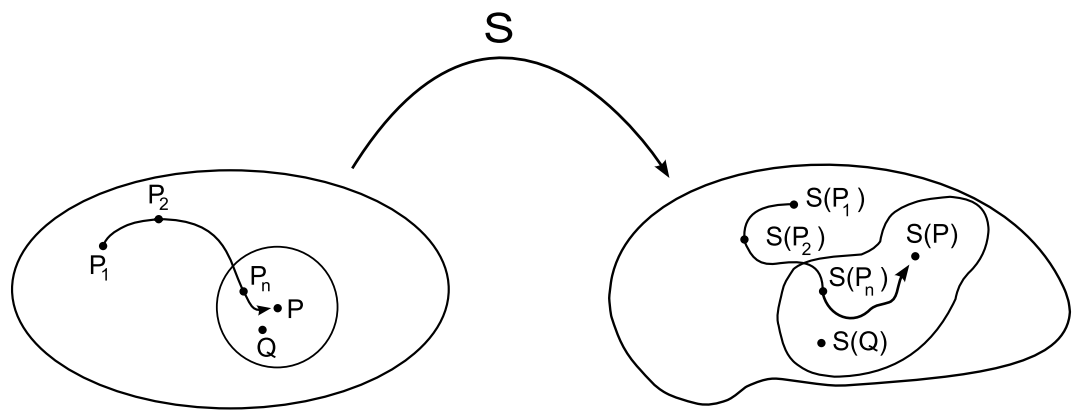




\section{Derivatives and Influence Functions}

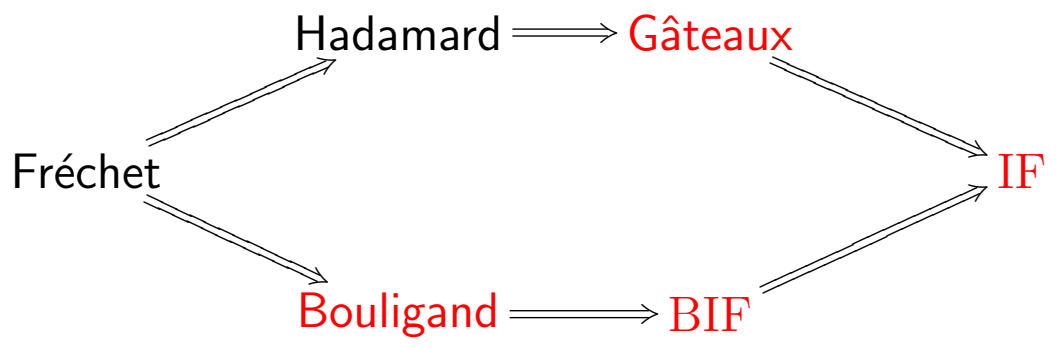

Christmann \& Van Messem (2008)

Notation: $\nabla^{F}, \nabla^{G}, \nabla^{B}, \nabla_{3}^{B}$, etc.

Property: $\nabla_{3}^{F} L^{\star}=\nabla_{3}^{F} L, \nabla_{3}^{B} L^{\star}=\nabla_{3}^{B} L$ 


\section{Bouligand differentiability}

\section{Bouligand-derivative}

$f: U \rightarrow Z$ is Bouligand-differentiable at $x_{0} \in U$, if $\exists$ a positive homogeneous function $\nabla^{B} f\left(x_{0}\right): U \rightarrow Z$ such that

$$
f\left(x_{0}+h\right)=f\left(x_{0}\right)+\nabla^{B} f\left(x_{0}\right)(h)+o(h),
$$

i.e.

$$
\lim _{h \downarrow 0} \frac{\left\|f\left(x_{0}+h\right)-f\left(x_{0}\right)-\nabla^{B} f\left(x_{0}\right)(h)\right\|_{Z}}{\|h\|_{U}}=0 .
$$

$g: E \rightarrow F$ positive homogeneous if

$$
g(\alpha x)=\alpha g(x) \quad \forall \alpha \geq 0, \forall x \in E
$$




\section{Influence Function}

\section{Definition (Hampel, '68, Hampel et al. '86)}

The influence function (IF) of a function $S: \mathcal{M}_{1} \rightarrow \mathcal{H}$ for a distribution $\mathrm{P}$ is given by

$$
\operatorname{IF}(z ; S, \mathrm{P}):=\lim _{\varepsilon \downarrow 0} \frac{S\left((1-\varepsilon) \mathrm{P}+\varepsilon \delta_{z}\right)-S(\mathrm{P})}{\varepsilon},
$$

in those $z:=(x, y) \in \mathcal{X} \times \mathcal{Y}$ where this limit exists.

If $\nabla^{G}(z ; S, \mathrm{P})$ exists: $\nabla^{G}=\mathrm{IF}$ and IF is linear and continuous

Goal: Bounded IF

Problem: Loss function $L$ often not Fréchet-differentiable 


\section{Bouligand Influence Function}

\section{Definition (C\&VM '08)}

The Bouligand influence function (BIF) of a function

$S: \mathcal{M}_{1} \rightarrow \mathcal{H}$ for a distribution $\mathrm{P}$ in the direction of a distribution $\mathrm{Q} \neq \mathrm{P}$ is the special Bouligand-derivative

$$
\lim _{\varepsilon \downarrow 0} \frac{\|S((1-\varepsilon) \mathrm{P}+\varepsilon \mathrm{Q})-S(\mathrm{P})-\mathrm{BIF}(\mathrm{Q} ; S, \mathrm{P})\|_{\mathcal{H}}}{\varepsilon}=0
$$

(if it exists).

If $\mathrm{BIF}$ exists and $\mathrm{Q}=\delta_{z}: \mathrm{IF}$ exists and $\mathrm{BIF}=\mathrm{IF}$

Goal: Bounded BIF 


\section{Result for BIF}

\section{Assumptions}

- $\mathcal{H}$ is RKHS with bounded, continuous kernel $k$

- $L$ convex and Lipschitz continuous with $|L|_{1} \in(0, \infty)$

- $\nabla_{3}^{B} L(x, y, \cdot)$ and $\nabla_{3,3}^{B} L(x, y, \cdot)$ measurable with

$$
\begin{aligned}
& \kappa_{1}:=\sup _{(x, y) \in \mathcal{X} \times \mathcal{Y}}\left\|\nabla_{3}^{B} L(x, y, \cdot)\right\|_{\infty} \in(0, \infty), \\
& \kappa_{2}:=\sup _{(x, y) \in \mathcal{X} \times \mathcal{Y}}\left\|\nabla_{3,3}^{B} L(x, y, \cdot)\right\|_{\infty}<\infty
\end{aligned}
$$

- $\lambda>\frac{1}{2} \kappa_{2}\|k\|_{\infty}^{3} \quad\left(\kappa_{2}=0\right.$ for eps-insensitive and pinball $)$

- $\mathrm{P} \neq \mathrm{Q}$, probability measures on $\mathcal{X} \times \mathcal{Y}$ 


\section{Theorem BIF}

Then $\operatorname{BIF}(\mathrm{Q} ; S, \mathrm{P})$ with $S(\mathrm{P}):=f_{L^{\star}, \mathrm{P}, \lambda}$ and $\mathrm{Q} \neq \mathrm{P} \in \mathcal{M}_{1}$

(1) exists,

(2) equals

$$
\begin{aligned}
T^{-1} & \left(\mathbb{E}_{\mathrm{P}} \nabla_{3}^{B} L^{\star}\left(X, Y, f_{L^{\star}, \mathrm{P}, \lambda}(X)\right) \Phi(X)\right. \\
& \left.-\mathbb{E}_{\mathrm{Q}} \nabla_{3}^{B} L^{\star}\left(X, Y, f_{L^{\star}, \mathrm{P}, \lambda}(X)\right) \Phi(X)\right),
\end{aligned}
$$

where $T: \mathcal{H} \rightarrow \mathcal{H}$ with $T(\cdot):=$

$2 \lambda \operatorname{id}_{\mathcal{H}}(\cdot)+\mathbb{E}_{\mathrm{P}} \nabla_{3,3}^{B} L^{\star}\left(X, Y, f_{L^{\star}, \mathrm{P}, \lambda}(X)\right)\langle\Phi(X), \cdot\rangle_{\mathcal{H}} \Phi(X)$,

(3) is bounded. 


\section{Simulated data}

- Predict $f(x)=50 \sin (x / 20) \cos (x / 10)+x$

- $n=1000$ data points $x_{i} \sim \mathcal{U}(-100,100)$

- Output $y_{i}=f\left(x_{i}\right)+\varepsilon_{i}$, where $\varepsilon_{i} \sim$ Cauchy distribution

- $\epsilon$-insensitive loss and Gaussian RBF kernel

- hyperparameters $(\lambda, \epsilon, \gamma)$ determined by minimizing $L^{\star}$-risk via grid search over $17 \times 12 \times 17=3468$ knots

- $\lambda$ regularization parameter of SVM

- $\epsilon$ parameter of $\epsilon$-insensitive loss

- $\gamma$ parameter of Gaussian RBF kernel

Result $(\lambda, \epsilon, \gamma)=\left(2^{-12}, 2^{-8}, 2^{-4}\right)$ 


\section{Simulated data}

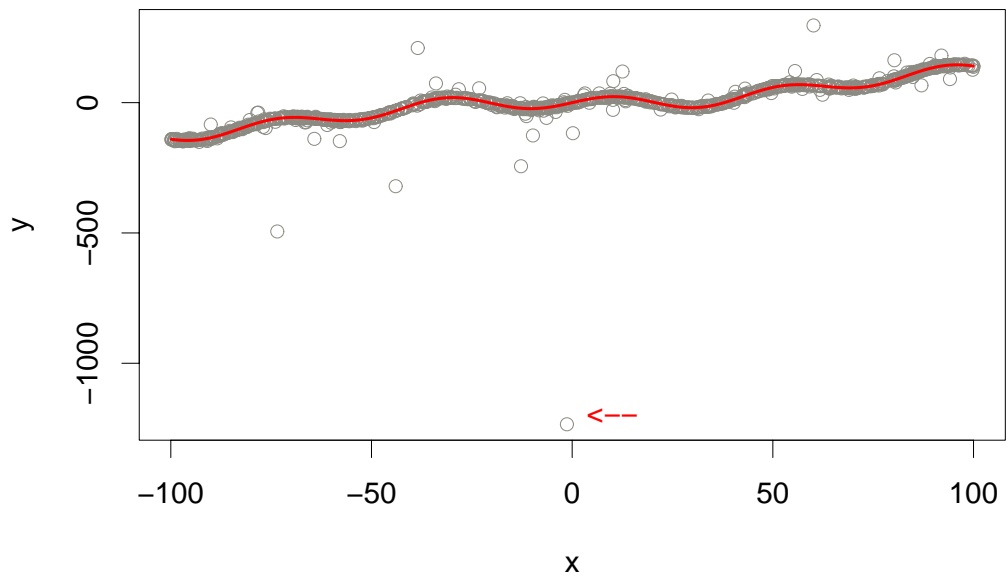




\section{Simulated data}

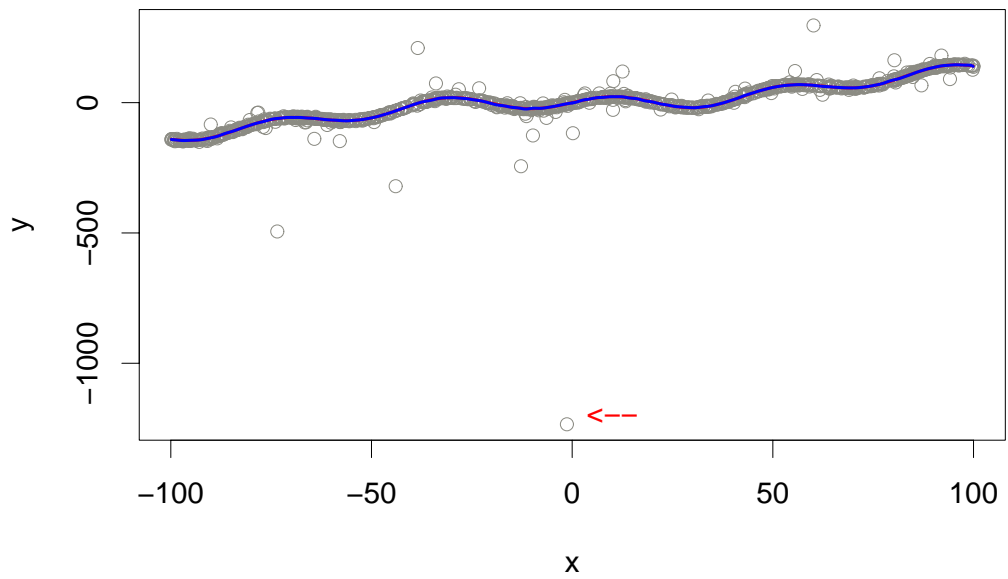




\section{Simulated data}

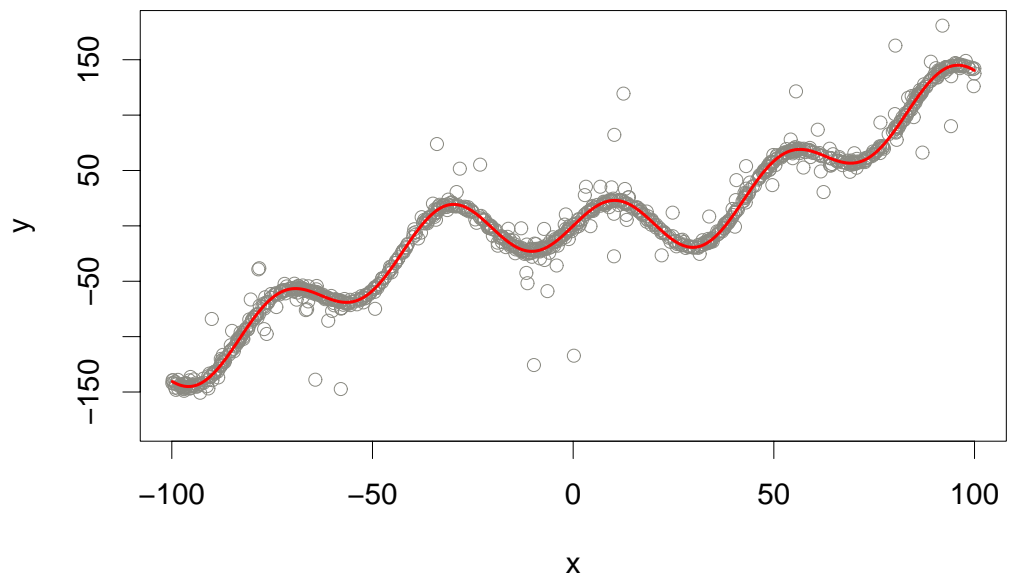




\section{Simulated data}

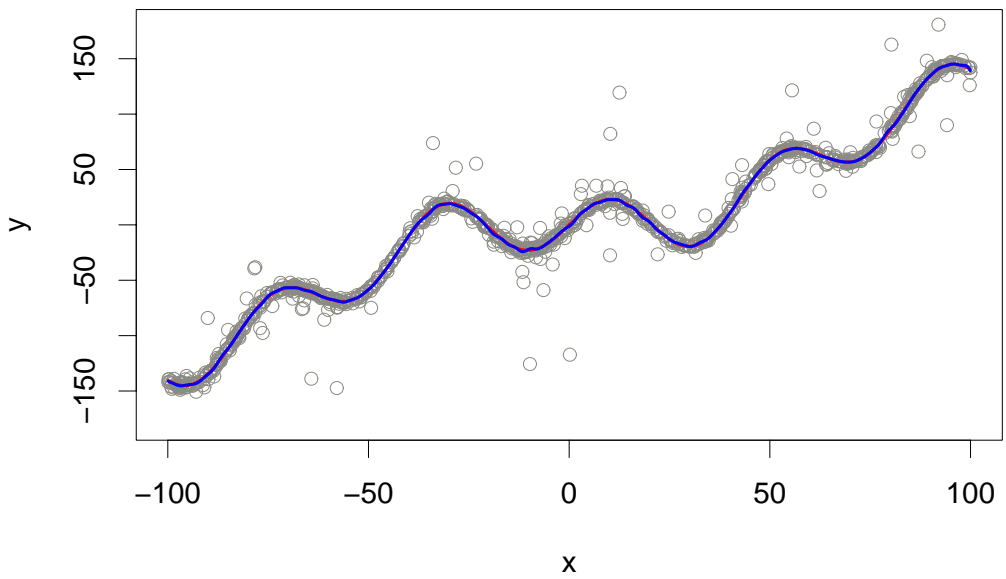




\section{Danish data}

- 2167 fire insurance claims over 1 million DKK (1980 1990)

- Regression with time as explanatory variable

- Classical least squares regression

- Conditional quantile regression using SVMs

- Pinball loss for $\tau \in\{0.50,0.75,0.90,0.99,0.995\}$

- Gaussian RBF kernel

- Extreme value distribution 


\section{Danish data}

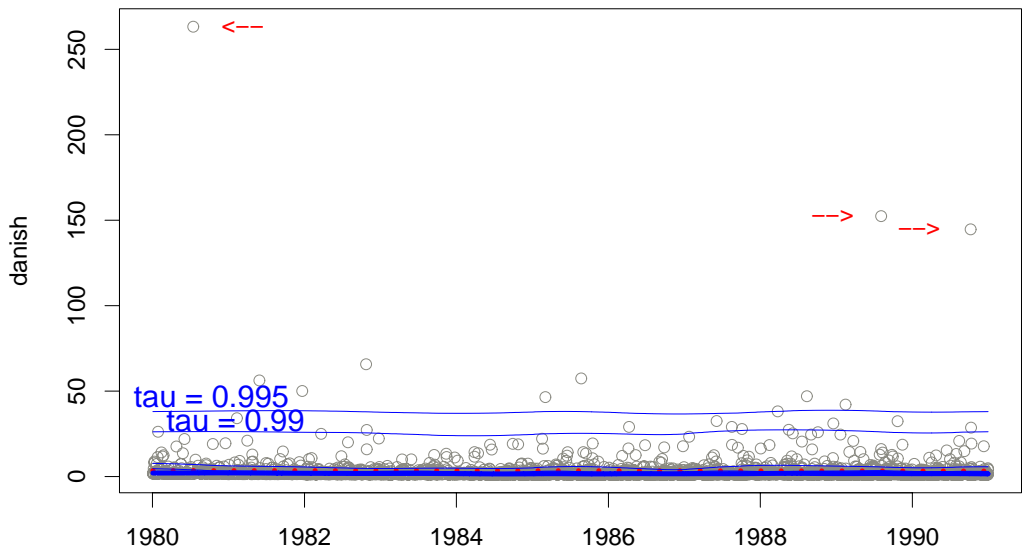




\section{Danish data}

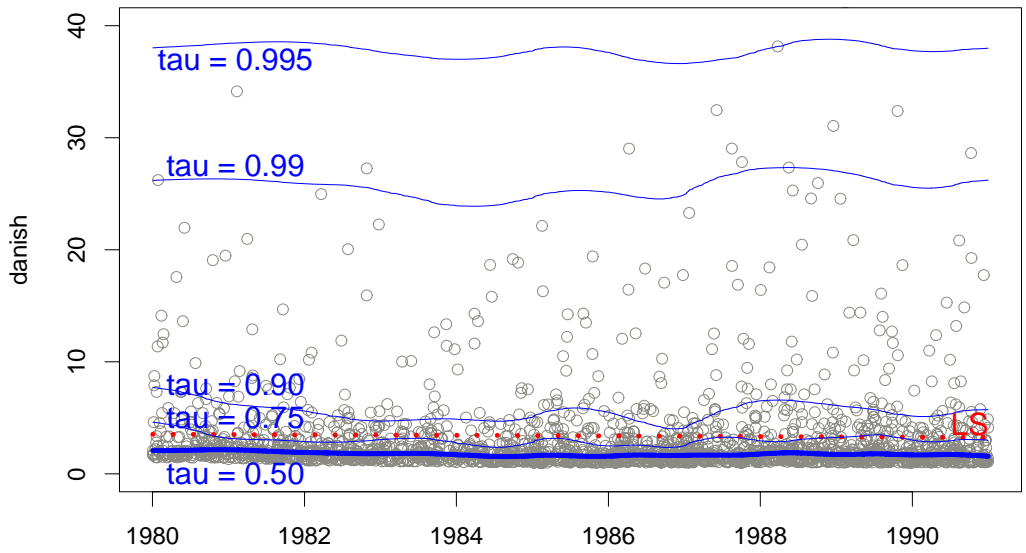




\section{Danish data}

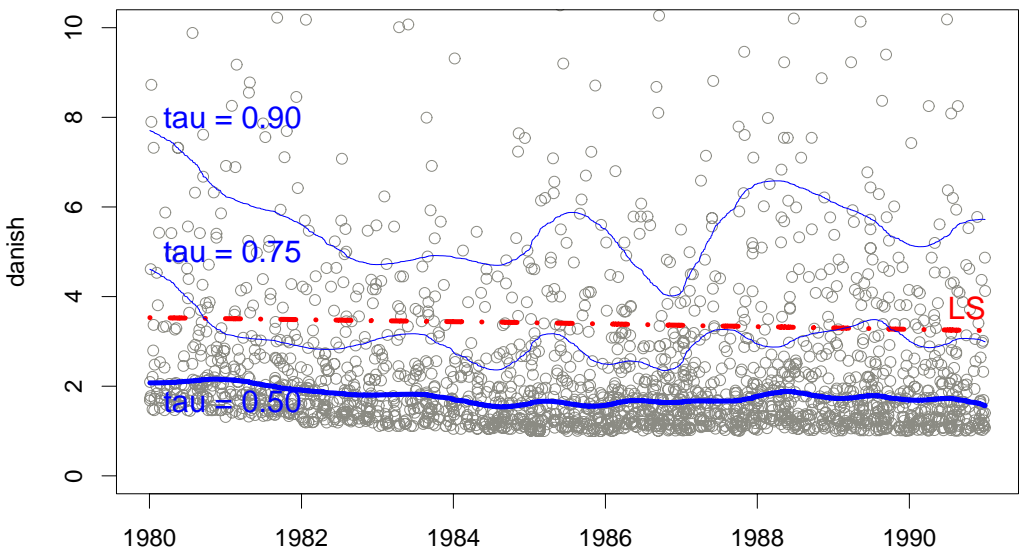




\section{Conclusions}

SVMs based on $L^{\star}(x, y, t):=L(x, y, t)-L(x, y, 0)$

(1) Weaker assumption on $\mathrm{P}$ : only $f \in L_{1}\left(\mathrm{P}_{X}\right)$ is needed e.g. $f$ bounded and $\mathcal{X} \subset \mathbb{R}^{d}$ bounded

(2) Existence and uniqueness of $f_{L^{\star}, \mathrm{P}, \lambda}$

(3) Consistency of risk and SVM solution

(4) Robustness

- Existence of BIF

- $\operatorname{BIF}(\mathrm{Q} ; S, \mathrm{P})$ bounded if $\nabla_{3}^{B} L, \nabla_{3,3}^{B} L$ measurable and bounded as well as $k$ continuous and bounded 


\section{References}

- Van Messem \& Christmann (2010). Advances in Data Analysis and Classification, accepted.

- Christmann, Van Messem \& Steinwart (2009). Statistics and Its Interface, 2, 311-327.

- Christmann \& Van Messem (2008). Journal of Machine Learning Research, 9, 915-936.

- Steinwart \& Christmann (2008). Support Vector Machines. Springer, New York.

- Christmann \& Steinwart (2007). Bernoulli, 13, 799-819.

- Hampel (1974). J. Amer. Statist. Assoc., 69, 383-393.

- Huber (1967). Proceedings of the $5^{\text {th }}$ Berkeley Symposium.

- Koenker (2005). Quantile regression. Cambridge University Press.

- Schölkopf \& Smola (2002). Learning with kernels. MIT Press.

- Vapnik (1998). Statistical learning theory. Wiley. 


\section{Reason}

\section{Conditions for finite risk}

For $L$ Lipschitz continuous

- $\mathbb{E}_{\mathrm{P}} L(X, Y, f(X))<\infty$ if $f \in L_{1}\left(\mathrm{P}_{X}\right)$ and $Y \in L_{1}\left(\mathrm{P}_{Y \mid x}\right)$.

$\mathcal{R}_{L, \mathrm{P}}(f) \leq|L|_{1}\left(\int_{\mathcal{X}}|f(x)| d \mathrm{P}_{X}(x)+\int_{\mathcal{X}} \int_{\mathcal{Y}}|y| d \mathrm{P}(y \mid x) d \mathrm{P}_{X}(x)\right)$

- $\mathbb{E}_{\mathrm{P}} L^{\star}(X, Y, f(X))<\infty$ if $f \in L_{1}\left(\mathrm{P}_{X}\right)$.

$$
\mathcal{R}_{L, \mathrm{P}}(f) \leq|L|_{1} \int_{\mathcal{X}}|f(x)| d \mathrm{P}_{X}(x)
$$

